\title{
Determining the Orientation of a Chiral Substrate Using Full-Hemisphere Angle-Resolved Photoelectron Spectroscopy
}

\author{
A. Tadich, ${ }^{1,2}$ J. Riley, ${ }^{2}$ L. Thomsen, ${ }^{1,3}$ B. C. C. Cowie, ${ }^{1}$ and M. J. Gladys ${ }^{3}$ \\ ${ }^{1}$ Australian Synchrotron, Clayton, VIC 3168, Australia \\ ${ }^{2}$ Department of Physics, La Trobe University, Bundoora, VIC 3083, Australia \\ ${ }^{3}$ School of Mathematical and Physical Sciences, University of Newcastle, Callaghan, NSW 2308, Australia \\ (Received 17 June 2011; revised manuscript received 29 August 2011; published 17 October 2011)
}

Chiral interfaces and substrates are of increasing importance in the field of enantioselective chemistry. To fully understand the enantiospecific interactions between chiral adsorbate molecules and the chiral substrate, it is vital that the chiral orientation of the substrate is known. In this Letter we demonstrate that full-hemisphere angle-resolved photoemission permits straightforward identification of the orientation of a chiral surface. The technique can be applied to any solid state system for which photoemission measurements are possible.

DOI: 10.1103/PhysRevLett.107.175501

PACS numbers: 61.05.js, 71.18.+y, 79.60.-i

Any two objects which are nonsuperimposable mirror images of one another are chiral; this property is widely exhibited in nature, particularly in the biological realm. Materials that exhibit a chiral or nonsymmetric surface structure have the potential to play a significant role as environments for enantioselective adsorption, permitting for instance, heterogeneous catalysis of small enantiopure molecules. Such materials include in situ grown $\mathrm{Cu}$ oxides [1], surfaces templated with chiral molecules [2], and certain high Miller index body- centered cubic (bcc) and face- centered cubic (fcc) metal surfaces [3-5]. The latter surfaces have seen increased attention due to their reactivity and ability to distinguish between two chiral enantiomers [6]. In general, the surface of a fcc material will be chiral provided that its Miller indices $\{h k l\}$ satisfy the constraints $h \neq k \neq l$ and $h k l \neq 0$. These surfaces contain kink atoms that are formed by the intersection of $\{111\}$, $\{110\}$ and $\{100\}$-oriented microfacets, with the rotational order of the microfacets used as a basis for labelling of the chiral orientation [7]. An example is shown in Fig. 1(a), for the chiral surfaces $\mathrm{Cu}\{421\}^{R}$ and $\mathrm{Cu}\{421\}^{S}$. Here, the kink atom is viewed from above, and the microfacets intersecting at the site are given an order of priority $\{111\}>\{110\}>\{100\}$ based on the atomic densities of the planes. If this sequence runs clockwise or anticlockwise the surface is denoted " $R$ " or " $S$," respectively.

A critical aspect of enantiospecific experiments is knowledge of the chiral orientation of the surface. Conventional (single energy) low energy electron diffraction (LEED) can be used to determine the orientation of many of these surfaces; however, it is not possible for those surfaces possessing an achiral unit cell (e.g., the fcc $\{421\}$ and fcc $\{531\}$ systems) [3]. This can be overcome with a more complex LEED-IV experiment; recent studies on $\mathrm{Cu}\{531\}$ and $\mathrm{Pt}\{531\}$ implemented a detailed experimental and theoretical LEED-IV analysis of the clean $R$ and $S$ surfaces, yielding the absolute chiral orientation in both cases [8,9]; however, the analysis is quite involved. X-ray diffraction (XRD) also permits identification of chiral orientation; however, this is not a typical instrument available inside modern ultra high vacuum (UHV) surface science chambers, and is instead done ex situ[3]. For novel systems that are prepared in situ in the UHV environment, the range of options for determining the chiral orientation is limited. Surface science techniques such as photoelectron spectroscopy have so far proved invaluable in probing the interaction of chiral molecules on surfaces [10,11], and thus it is natural to ask whether photoemission itself can reveal the absolute chirality of the surface. The photoelectron angular emission distribution is intrinsically related to molecular and crystalline symmetry; in principle, the complete fullhemisphere (or " $2 \pi$ ") photoelectron emission from a sample should yield details of the chiral orientation. Fullhemisphere angle-resolved photoemission measurements are usually applied in two distinct experimental techniques: x-ray photoelectron diffraction (XPD) and Fermi surface (FS) mapping [12-14]. In the context of chiral systems only a few studies exist in which full-hemisphere photoemission has been applied, using XPD to investigate the local absorption geometry of chiral molecules onto chiral or achiral substrates. For example, Greber et al measured full-hemisphere XPD from $R$ and $S$ cysteine adsorbed on $\operatorname{Au}(17119)$, in which the chirality of the substrate was already known [15]. Fasel et al investigated the absorption of heptahelicene on $\mathrm{Cu}(111)$ and $\mathrm{Cu}(332)$, utilizing XPD to characterize the geometrical orientation of this chiral molecule on the achiral substrates [16]. In this Letter we demonstrate that the absolute orientation of a chiral single crystal surface can be determined using either full-hemisphere core level XPD or valence band FS mapping. We show that in each case the emission distribution is a straightforward reflection of the symmetry of the underlying lattice, leading to an unambiguous assignment of the chiral orientation. 
(a)

$\mathrm{Cu}\{421\}^{S}$
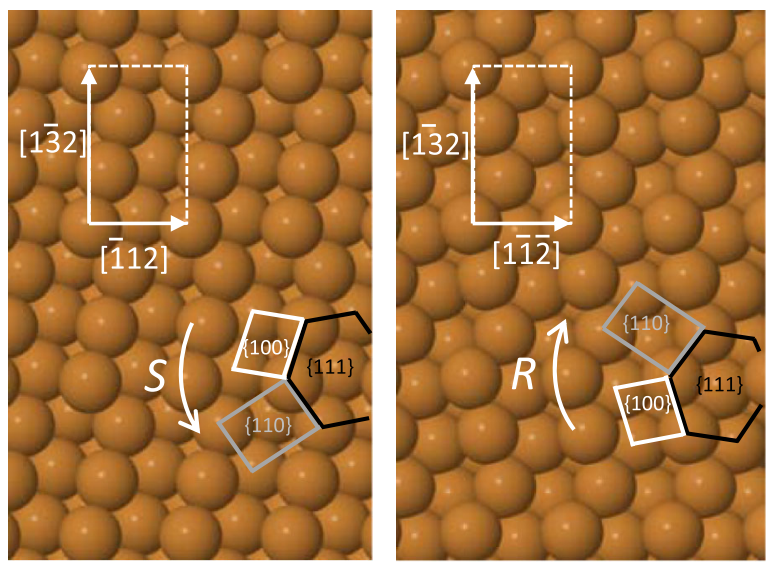

(b) Projection of Low-index planes and directions
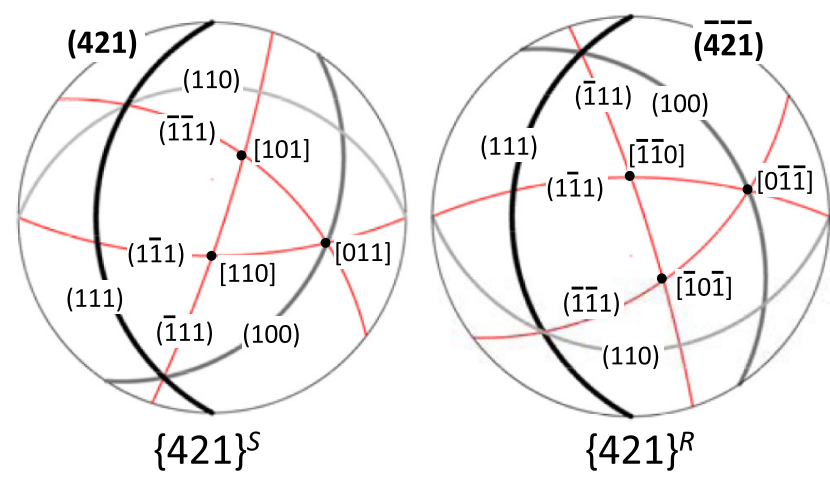

(c)

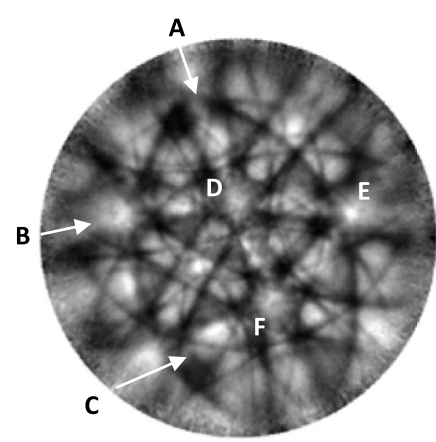

XPD Experiment $+\{421\}^{R}$ planes

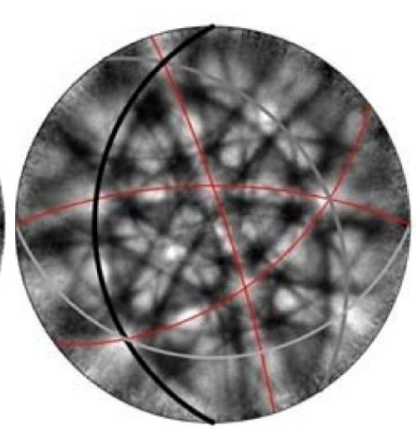

FIG. 1 (color online). (a) Hard sphere models of the $\mathrm{Cu}\{421\}^{S}$ (left) and $\mathrm{Cu}\{421\}^{R}$ (right) surfaces. The surface unit cell and directions are indicated. The $\{111\},\{100\}$ and $\{110\}$ microfacets are shown (black, dark grey and light grey, respectively). (b) Stereographic projection of the key low-index planes and directions onto each surface portrayed in Fig. 1(a); the projection of the (111), (100) and (110) planes are shown in black, dark grey and light grey, respectively. Three nonequivalent planes from the $\{111\}$ family are shown as thin red [medium gray] lines. (c) Experimental XPD data (left) $(h \nu=600 \mathrm{eV})$ shown in stereographic projection. Features $A$ to $E$ are ascribed to key forward focussing structures, and are described in the Letter. The right-hand side overlays the stereographic projection of planes associated with the $\{421\}^{R}$ surface as shown in Fig. 1(b).
The angle-resolved photoemission experiments were performed on a prototypical chiral surface, $\mathrm{Cu}\{421\}$, using the toroidal angle-resolving electron spectrometer located at beam line UE56/2-PGM1, BESSY [17]. The fullhemisphere photoemission measurements were performed by stepping the sample's azimuthal angle and measuring over all polar emission angles, resulting in a dense sampling mesh ( $>65 k$ points) of emission directions from the surface. The electron analyzer was adjusted in each case to collect electrons from an energy window centered at the relevant initial state. The XPD measurement was carried out by measuring the $\mathrm{Cu} 3 p_{3 / 2}$ core level excited with a photon energy of $h \nu=600 \mathrm{eV}$, while the FS data were obtained at $h \nu=200 \mathrm{eV}$ using electrons excited from the Fermi energy.

We begin by discussing the results of the XPD experiment. The left-hand side of Fig. 1(c) shows the experimental results, plotted as an intensity modulation function using a smooth polar-angle dependant background as described by Fasel et al [16]. The data is plotted as a stereographic projection, with the center of the plot corresponding to normal emission, and the edges to grazing emission, from the surface. The plot shows strong intensity variations in the azimuthal and polar emission angles. For example, three distinct bands of intensity traverse the data (lines $A, B$ and $C$ ). At the three points at which these bands cross, a clear enhancement in intensity is observed (points $D, E$, and $F$ ), forming the vertices of a downward-pointing triangle. The origin of these features in XPD is well understood for systems studied with photoelectron energies greater than $500 \mathrm{eV}$ [18-21]. The differential elastic scattering cross section for the photoelectrons is dominated in this instance by the forward-scattering component, resulting in a "forward focussing effect" in which the photoemission intensity is enhanced along low-index planes and directions of high atomic density. The intensity distribution shown in Fig. 1(c) obtained at $h \nu=600 \mathrm{eV}$ can essentially be interpreted as the projection of the bulk geometry of the underlying copper lattice onto the surface. To understand which key crystal directions contribute to the data, we consider the stereographic projection of low-index planes and directions for the ideal $\mathrm{Cu}\{421\}^{S}$ and $\mathrm{Cu}\{421\}^{R}$ surfaces. The use of stereographic projections in describing the symmetry and orientation of chiral surfaces from fcc and bcc lattices is well known, and has recently been reviewed by Jenkins and Pratt [22], Hard sphere models of both surfaces are shown in Fig. 1(a). The stereographic projection of the (111), (100), and (110) planes (black, dark grey, and light grey, respectively) onto each surface are shown in Fig. 1(b), as well as the projection of three planes from the $\{111\}$ family: (111), (1 $\overline{1} 1)$, and $(\overline{1} \overline{1} 1)$. The intersection of neighboring pairs of planes from this latter set correspond to low-index directions from the $\{110\}$ family; these are indicated as black circles. The data clearly shows structure related to these 
features, but importantly a closer inspection and comparison with the calculated projections for each surface reveals that the relative orientation of features in the data is only compatible with those for the $R$ surface. Features $A$, $B$, and $C$ agree exactly with the $\{111\}$-type plane projections (red [medium gray] lines), and $D, E$, and $F$ represent the strong forward focussing along the $\{110\}$-oriented atomic chains. It is impossible to rotate the data (a symmetry preserving operation) such that points $D, E$, and $F$ form an upward pointing triangle, with features $A$, $B$, and $C$ oriented in the correct way, in order to match the geometrical projection for the $S$ surface. The exact agreement with the $R$ surface is highlighted on the right side of Fig. 1(c), where the calculated projections are overlaid onto the data. The results suggest that since high energy full-hemisphere XPD directly yields a bulk projection of real-space structure through the crystal surface, the extension of the method to determine the orientation of more complex chiral structures such as in situ grown layers is straightforward. One only requires a model of the ideal real-space geometry for the pair of opposing chiral surfaces, which can be directly compared with the experiment; it would be applicable, for example, to chiral facets of quartz, which can be grown in UHV on suitable substrates as bulklike thin film layers [23-25]. Comparison of the XPD data with a series of stereographic projections of the known bulk lattice would lead to the identification of the orientation of the film.

The complementary results and calculations for the FS experiment are shown in Fig. 2. The absorption of photons at the Fermi level results in energy and wave-vector $(\boldsymbol{k})$ conserving transitions to unfilled final states above the vacuum level [26]. The component of photoelectron wave vector parallel to the crystal surface is conserved upon emission into the vacuum, and the symmetry of the external wave-vector distribution will equal the symmetry of the underlying lattice with respect to the surface. This is expected to hold true even for these chiral surfaces, which are known to exhibit surface roughening and faceting [27-29]. Figure 2(a) shows the experimental FS data taken at $h \nu=200 \mathrm{eV}$, in which the full-hemisphere wave-vector distribution has been resolved into orthogonal components in the surface plane. Intense and sharp contours correspond to direct transitions from the copper FS, with the origin of these features being well understood [30-32]. Two salient features are highlighted for discussion. Feature 1 represents the sampling of the final state across four FS bodies, resolving the well-known FS "dogsbone" for copper [33]. Feature 2, a flowerlike contour with a circular structure at the center, corresponds to the final-state cutting across the FS "neck" along a $\{111\}$ direction. A comparison of the data can be made with a simple approximation of direct transitions from the FS, the so-called free electron final state (FEFS), which is a valid model at the photon energy used here [26]. The results of a FEFS calculation for the $S$ (a) FS Experiment $(h v=200 \mathrm{eV})$

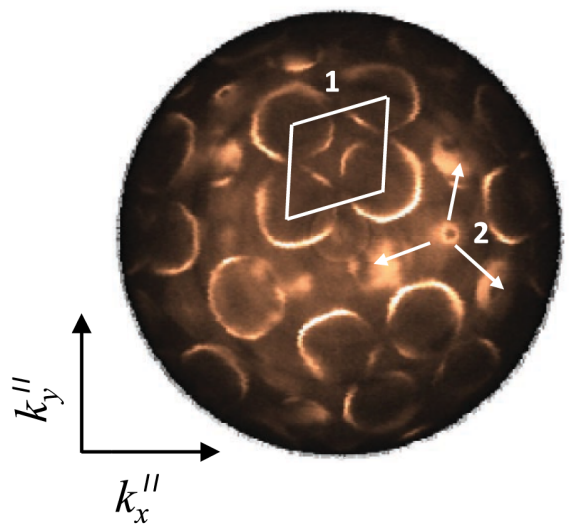

(b) FEFS calculations
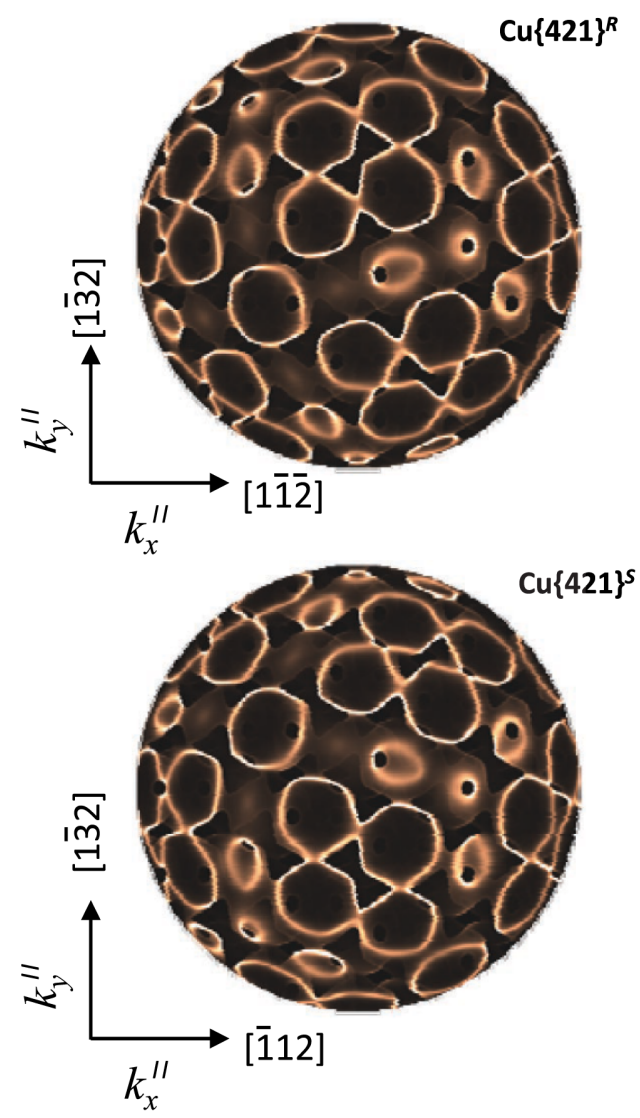

FIG. 2 (color online). (a) Full-hemisphere FS data taken on $\mathrm{Cu}\{421\}(h \nu=200 \mathrm{eV}) ; k_{x}^{\|}$and $k_{y}^{\|}$represent the surfaceresolved components of the vacuum wave vector. Features 1 and 2 represent key structures that are discussed accordingly in the Letter. (b) Free electron final-state (FEFS) calculation of the fullhemisphere emission from the FS for the $\mathrm{Cu}\{421\}^{R}$ and $\mathrm{Cu}\{421\}^{S}$ surfaces $(h \nu=200 \mathrm{eV}$, Inner Potential $=12 \mathrm{eV})$. The $k^{\|}{ }_{x}$ and $k^{\|}{ }_{y}$ surface directions are indicated for each calculation.

and $R$ surfaces is shown in Fig. 2(b); the calculation also incorporates $k$-space broadening due to the small inelastic mean free path of the electrons [34]. The FEFS approximation has been applied to a density functional theory 
(DFT) band structure calculation for copper. Within the calculations for both surfaces, one can identify spectral structures closely resembling features 1 and 2. Clearly, there exists discrepancy in the intensity between the experimental and calculated data; however, the FEFS is a simple model of the photoemission process, and does not contain photoemission matrix elements. However, any intensity disagreements are of minor importance as it is the symmetry and position of the direct transition features that will reveal the chiral orientation. In a similar manner to the XPD analysis, comparison of the calculations with the data reveals that the relative orientation of features 1 and 2 as calculated for the $S$ surface do not match those measured in the experiment. On the other hand, the data is in excellent agreement with the calculation from the $R$ surface, and the chirality is unambiguously determined. Full-hemisphere emission distributions from the valence band can also be calculated with the use of photoemission codes [13]. Hence, for applications involving novel chiral substrates of unknown orientation, calculations can be compared against a full-hemisphere valence band measurement in order to deduce the chiral orientation, in a manner similar to that described for the XPD analysis.

In conclusion, we have demonstrated that the absolute orientation of a chiral $\mathrm{Cu}\{421\}$ single crystal surface can be determined in a straightforward manner using fullhemisphere angle-resolved photoemission. From the XPD collected at relatively high electron energies, the orientation of the chiral substrate is revealed via careful comparison of the data with the stereographic projection of the real-space lattices of the two possible chiral structures. As an alternative technique, full-hemisphere FS mapping is able to reveal the orientation by a direct comparison of the data with a model of photoemission from the FS, applied to an appropriate electronic structure calculation. The application of these methods to elucidate the chiral orientation of more complex and novel chiral systems, especially those grown in situ in a vacuum environment is straightforward, provided the system is amenable to photoemission.

We acknowledge BESSY II (Helmholtz-Zentrum Berlin) for provision of synchrotron radiation at beam line UE56/2 PGM1, and would like to thank the beam line support staff for technical assistance. We acknowledge travel funding provided by the International Synchrotron
Access Program (ISAP) managed by the Australian Synchrotron and funded by the Australian Government.

[1] E. W. Bohannan et al., J. Am. Chem. Soc. 126, 488 (2004).

[2] K. H. Ernst et al., Nanotechnology 10, 355 (1999).

[3] D. M. Rampulla et al., J. Phys. Chem. B 110, 10411 (2006).

[4] C. Bombis et al., ACS Nano 4, 297 (2010).

[5] J. D. Horvath and A. J. Gellman, Top. Catal. 25, 9 (2003).

[6] G. Held and M. J. Gladys, Top. Catal. 48, 128 (2008).

[7] A. Ahmadi et al., Langmuir 15, 2420 (1999).

[8] G. Jones et al., Phys. Rev. B 79, 165420 (2009).

[9] S. R. Puisto et al., J. Phys. Chem. B 109, 22456 (2005).

[10] L. Thomsen et al., Surf. Sci. 603, 1253 (2009).

[11] M. J. Gladys et al., J. Phys. Chem. C 111, 8331 (2007).

[12] M. C. Asensio et al., Phys. Rev. B 67, 4519 (2003).

[13] M. Lindroos and A. Bansil, Phys. Rev. Lett. 77, 2985 (1996).

[14] P. Aebi et al., Phys. Rev. Lett. 72, 2757 (1994).

[15] T. Greber et al., Phys. Rev. Lett. 96, 056103 (2006).

[16] R. Fasel et al., J. Chem. Phys. 115, 1020 (2001).

[17] L. Broekman et al., J. Electron Spectrosc. Relat. Phenom. 144, 1001 (2005).

[18] T. Greber et al., Phys. Rev. Lett. 69, 1947 (1992).

[19] J. Osterwalder et al., Phys. Rev. B 44, 13764 (1991).

[20] D. Naumovic et al., Phys. Rev. B 47, 7462 (1993).

[21] L. Despont et al., Surf. Sci. 600, 380 (2006).

[22] S. J. Jenkins and S. J. Pratt, Surf. Sci. Rep. 62, 373 (2007).

[23] R. M. Hazen and D. S. Sholl, Nature Mater. 2, 367 (2003).

[24] T. Schroeder et al., Solid State Electron. 45, 1471 (2001).

[25] K. Soai et al., J. Am. Chem. Soc. 121, 11235 (1999).

[26] S. Hüfner, Photoelectron Spectroscopy: Principles and Applications (Springer-Verlag, Berlin, 2003).

[27] M. L. Clegg et al., J. Phys. Chem. C 114, 4114 (2010).

[28] R. Matzdorf, Surf. Sci. Rep. 30, 153 (1998).

[29] F. Baumberger, T. Greber, and J. Osterwalder, Phys. Rev. B 64, 195411 (2001).

[30] P. Aebi et al., Surf. Sci. 307, 917 (1994).

[31] A. P. J. Stampfl et al., Surf. Sci. 331, 1272 (1995).

[32] Z. Qu et al., Surf. Sci. 324, 133 (1995).

[33] J. A. Con Foo, Phys. Rev. B 53, 9649 (1996).

[34] V. N. Strocov, J. Electron Spectrosc. Relat. Phenom. 130, 65 (2003). 